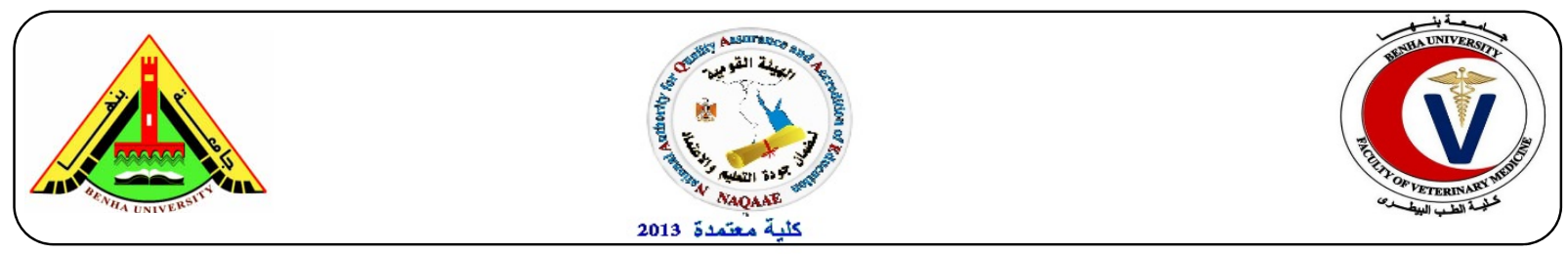

\title{
Effect of rosemary and green tea extracts on overall quality and shelf-life of meat during storage.
}

\author{
Amani, M. Salem ${ }^{1}$, Dalia, F. Khater ${ }^{2}$ and Shimaa, A. El Barody. \\ ${ }^{1}$ Food Control Dept., Faculty of Veterinary Medicine, Benha University, Egypt. \\ ${ }^{2}$ Animal Health Research Institute., Department of Food Hygiene., Tanta branch.
}

\begin{abstract}
A B S T R A C T
The present study was designed to investigate antimicrobial and antioxidant activity of green tea and rosemary extracts on minced meat samples. Minced meat was divided into 2 groups, untreated (control) and treated samples. The treated samples homogenized with green tea extract $(700 \mathrm{ppm})$, green tea extract $(1000 \mathrm{ppm})$, rosemary extract $(0.2 \%)$, mixture of green tea extract $(500 \mathrm{ppm}) \&$ rosemary extract $(0.2 \%)$, and mixture of green tea extract $(500 \mathrm{ppm}) \&$ rosemary extract $(0.3 \%)$. The minced meat samples were examined physically, bacteriologically and chemically to determine their keeping quality by counting aerobic plate, Staphylococcal and Enterobacteriacae counts, as well as measuring pH, TBA and TVN immediately after preparation (zero time) to 6 days of chill storage $\left(4 \pm 1^{\circ} \mathrm{C}\right)$. Green tea $(1000 \mathrm{ppm})$ group give the best effectiveness with a significant advantage in extend shelf-life of refrigerated minced meat to 6 days compared to all groups specially control ones (3 days).
\end{abstract}

Key words: Antimicrobial, Antioxidant, Green tea, Rosemary.

(http://www.bvmj.bu.edu.eg)

(BVMJ-31(1): 17-23, 2016)

\section{INTRODUCTION}

Meat is an important source for some micronutrients such as trace elements and vitamins. In addition, meat as a protein-rich and carbohydrate-low product contributes to a low glycemic index, which is assumed to be beneficial with respect to obesity, diabetes development and cancer (Biesalski, 2005). However, meat get easily contaminated by pathogenic microorganisms present in animal prior to slaughter. It is therefore important to make meat safe for consumers in terms of stability, transportation and storage. Shelflife and maintenance of meat quality are influenced by a number of interrelated factors including holding temperature, which can result in determinable changes in the quality attributes of meat. Spoilage by microbial growth is the most important factor in relation to keeping quality of meat (Lambert et al., 1991). Moreover, spoilage of meat has remained a serious challenge in developing countries, including Egypt for decades, this has been due to poor storage systems in such countries where necessary facilities that could help to promote preservation are unavailable. Wherever, in most developing countries fresh meat forms a significant proportion of meat intake (Olaoye and Onilude, 2010). Although several synthetic food additives have been widely used in the meat industry to extend food shelf-life, inhibit lipid oxidation and delay or inhibit the growth of pathogenic microorganisms, the trend is to decrease their use because of the growing concern among consumers about such chemical additives. Consequently, search for natural additives, especially of plant origin, has notably increased in recent years indicating that application of natural food additives possessing both antioxidant and antimicrobial activities may be useful for maintaining meat quality, extending shelf-life and preventing economic loss (Mielnik et al., 2008). Green tea is one of the natural preservatives applied to many foods as antioxidant and antimicrobial agents (Kristanti and Punbusayakul, 2009). Rosemary have antimicrobial activity against both Gram-positive and Gram-negative bacteria due to the presence of phenolic compounds, carnosic acid and carnosol (Del Campo et al., 2000). Most food borne illnesses of S. aureus outbreaks are result of production of heat stable toxins in the food which may lead to severe food poisoning outbreaks. (Ahmed, 1991). On the other, hand Enterobacteriaceae group has an epidemiological interest as some of its members 
are pathogenic and may result in serious infections and food poisoning. (Mercuri et al., 1978). Sensory, microbiological and biochemical methods have been used to assess freshness and quality during handling and storage with the main attributes of freshness being aroma, texture and appearance response (Koutsoumanis et al., 2002). Therefore, the present study was performed to investigate the antioxidant as well as the antimicrobial effectiveness of green tea and rosemary ethanolic extracts at various concentrations on the quality of fresh minced meat during chill storage $\left(4 \pm 1^{\circ} \mathrm{C}\right)$.

\section{MATERIAL AND METHODS}

\subsection{Collection of samples:}

About $5 \mathrm{~kg}$ minced meat samples were collected from different butchers shops from Tanta city and transported in an ice box as rapidly as possible to the laboratory. Minced meat samples were divided into 2 groups, untreated (control) and treated ones. The treated samples were homogenized with green tea extract $(700 \mathrm{ppm})$, green tea extract (1000 ppm), rosemary extract $(0.2 \%)$, mixture of green tea extract $(500 \mathrm{ppm}) \&$ rosemary extract $(0.2 \%)$, and mixture of green tea extract (500 ppm) \& rosemary extract $(0.3 \%)$. Both untreated and treated samples were packed in a separate plastic bags, stored at $\left(4 \pm 1^{\circ} \mathrm{C}\right)$ and then examined sensory, bacteriologically and chemically after (2 hrs) as zero time and at predetermined interval (24 hrs). Preparation of plant extracts according to(Gramza et al., 2004).

\subsection{Sensory analyses:}

The colour, odor and overall acceptability were determined for each sample of meat and were recommended by (Pearson and Tauber, 1984).

\subsection{Bacteriological analyses:}

Preparation of samples following (ICMSF, 1978) was applied as $10 \mathrm{~g}$ portion of each sample was aseptically weighed into $90 \mathrm{ml}$ of $0.1 \%$ peptone water in a sterile plastic bag, and then blended in a Stomacher 400 Lab Blender for 30 seconds. Ten-fold serial dilutions were used for bacteriological examination. Determination of Aerobic Plate Count according to ICMSF., (1996), Enterobacteriaceae count according to Gork, (1976), and Staphylococci count according to ICMSF., (1996).

\subsection{Chemical analyses:}

Determination of $\mathrm{pH}$ (Pearson and Tauber, 1984). Total Volatile Nitrogen (TVN) following Food and Agriculture Organization (FAO, 1980). and Thiobarbituric acid number (TBA) following (Vyncke, 1970). All experiments were conducted in triplicate.

\subsection{Statistical Analysis:}

ANOVA was carried out by SPSS version 16 on data of the sensory, chemical and microbiological evaluations. Data are expressed as mean \pm SD (Gomez and Gomez, 1984).

\section{RESULTS}

The results in table (1) showed that sensory analyses of control (untreated groups) remain accepted until $3^{\text {rd }}$ day while treated groups with green tea extract (700 ppm) and rosemary extract $(0.2 \%)$ remain accepted until $4^{\text {th }}$ day. Moreover, the present data mixture of green tea extract (500 ppm) \& rosemary extract $(0.2 \%)$, and mixture of green tea extract $(500 \mathrm{ppm}) \&$ rosemary extract $(0.3 \%)$ remain accepted until $5^{\text {th }}$ day as well as treated groups with green tea $(1000 \mathrm{ppm})$ extract still accepted until $6^{\text {th }}$ day. Table $(2 \& 3)$ indicated that the untreated samples showed the highest APC and Enterobacteriaceae counts (log. cfu/g) comparing to the treated ones. Both of APC and Enterobacteriaceae counts were gradually increased during cold storage for all samples with different ratios depending on the type and concentration of the extracts. The treated samples with green tea $(1000 \& 700 \mathrm{ppm})$ showed the lowest counts followed by mixture of green tea $(500 \mathrm{ppm}) \&$ rosemary extract $(0.3 \%)$ at all days of cold storage. The present data in table (4) showed results of Staphylococcus count (log. $\mathrm{cfu} / \mathrm{g}$ ) of treated and untreated minced meat groups showed significant differences during cold storage $\left(4 \pm 1^{\circ} \mathrm{C}\right)$. The treated groups with rosemary extract $(0.2 \%)(3.81 \pm 0.006)(\log$. cfu/g) were the lower while untreated groups were the highest at zero day $(4.57 \pm 0.02) \mathrm{cfu} / \mathrm{g}$. At $4^{\text {th }}$ day rosemary extract $(0.2 \%)$ followed by mixture of green tea extract $(500 \mathrm{ppm}) \&$ rosemary extract $(0.3 \%)$ and mixture of green tea extract $(500 \mathrm{ppm}) \&$ rosemary extract $(0.2 \%)(5.05 \pm 0.05,5.10 \pm 0.17,5.12 \pm 0.13)$ (log. cfu/g) were the lowest, while those treated with green tea extract $(700 \mathrm{ppm})$ and green tea extract $(1000 \mathrm{ppm})$ at $4^{\text {th }}$ day $(5.77 \pm 0.007$, $5.59 \pm 0.11) \quad(\log . \quad \mathrm{cfu} / \mathrm{g})$ were the highest, respectively.

Also, the results in table (5) showed that the reduction percentage of APC, Staphylococci and Enterobacteriaceae counts. The highest reduction percentage were in green tea extract (1000 ppm) which recorded $97 \%, 80 \%, 59 \%$ at the days of cold storage. It is evident from the present investigation 
that the differences in $\mathrm{pH}$ mean value between different treated and untreated samples were significant $(P<0.05)$ during storage at $\left(4 \pm 1^{\circ} \mathrm{C}\right)$ in table (6). The results showed an increase in $\mathrm{pH}$ mean values in untreated groups and decrease in treated ones. The highest rates were found in control samples $(6.0 \pm 0.14)$ while, treated samples with green tea $(700 \mathrm{ppm})$ were the lowest $(5.8 \pm 0.1)$ followed by mixture of green tea extract (500 ppm) $\&$ rosemary extract $(0.2 \%)(5.9 \pm 0.2)$, green tea $(1000 \mathrm{ppm})$, rosemary $(0.2 \%)$, mixture of green tea extract $(500 \mathrm{ppm}) \&$ rosemary extract $(0.3 \%)$ at $4^{\text {th }}$ day of cold storage at $\left(4 \pm 1^{\circ} \mathrm{C}\right)$, respectively. On the other hand, a significant $(P<0.05)$ increase in
TBA and TVN mean values in different treatments during storage by different rates shown in table (7\&8) The untreated (control) sample showed the highest value $(0.86 \pm 0.01) \mathrm{mg}$ Melanoaldehyde $/ \mathrm{Kg}$ in $2^{\text {nd }}$ day of storage, while the lowest values were recorded in samples treated with rosemary extract $(0.2 \%) \quad(0.64 \pm 0.01)$, also untreated (control) sample showed the highest value of TVN $(14.3 \pm 0.10) \mathrm{mg} / 100 \mathrm{~g}$ while rosemary $(0.2 \%)$ and mixture of green tea extract $(500 \mathrm{ppm}) \&$ rosemary extract $(0.3 \%)$ showed lowest value $(12.3 \pm 0.3)$, $(12.3 \pm 0.1) \mathrm{mg} / 100 \mathrm{~g}$, respectively at 2 nd day of cold storage.

Table (1) The effects of various concentrations of natural extracts on overall acceptability of the examined minced meat samples stored at $4 \pm 1{ }^{\circ} \mathrm{C}$.

\begin{tabular}{ccccccc}
\hline Days & $\begin{array}{c}\text { Control } \\
\text { sample }\end{array}$ & $\begin{array}{c}\text { Green tea } \\
(700 \mathrm{ppm})\end{array}$ & $\begin{array}{c}\text { Green tea } \\
(1000 \mathrm{ppm})\end{array}$ & $\begin{array}{c}\text { rosemary } \\
(0.2 \%)\end{array}$ & $\begin{array}{c}\text { Mixture of } \\
(500 \& 0.2 \%)\end{array}$ & $\begin{array}{c}\text { Mixture of } \\
(500 \& 0.3 \%)\end{array}$ \\
\hline zero & 9 & 9 & 9 & 9 & 9 & 9 \\
$1^{\text {st }}$ & 8 & 9 & 9 & 9 & 9 & 9 \\
$2^{\text {nd }}$ & 6 & 7 & 8 & 8 & 8 & 8 \\
$3^{\text {rd }}$ & - & 6 & 8 & 7 & 8 & 8 \\
$4^{\text {th }}$ & - & 5 & 7 & 6 & 7 & 7 \\
$5^{\text {th }}$ & - & - & 6 & - & 6 & 6 \\
$6^{\text {th }}$ & - & - & 5 & - & - & - \\
\hline
\end{tabular}

(9) Excellent, (8) Very very good, (7) Very good, (6) Good, (5) Medium

Table (2): The effects of various concentrations of natural extracts on APC (log. $\mathrm{cfu} / \mathrm{g})$ of the examined minced meat samples during cold storage at $4 \pm 1^{\circ} \mathrm{C}$.

\begin{tabular}{ccccccc}
\hline Days & Control & $\begin{array}{c}\text { Green tea } \\
(700 \mathrm{ppm})\end{array}$ & $\begin{array}{c}\text { Green tea } \\
(1000 \mathrm{ppm})\end{array}$ & $\begin{array}{c}\text { rosemary } \\
(0.2 \%)\end{array}$ & $\begin{array}{c}\text { Mixture of } \\
(500 \& 0.2 \%)\end{array}$ & $\begin{array}{c}\text { Mixture of } \\
(500 \& .03 \%)\end{array}$ \\
\hline Zero & $6.23 \pm 0.06^{\mathrm{a}}$ & $4.73 \pm 0.03^{\mathrm{d}}$ & $4.66 \pm 0.05^{\mathrm{d}}$ & $5.84 \pm 0.06^{\mathrm{b}}$ & $4.82 \pm 0.01^{\mathrm{c}}$ & $4.67 \pm 0.02^{\mathrm{d}}$ \\
$1^{\text {st }}$ & $6.38 \pm 0.06^{\mathrm{a}}$ & $5.63 \pm 0.30^{\mathrm{b}} \mathrm{c}$ & $5.41 \pm 0.39^{\mathrm{c}}$ & $5.95 \pm 0.04^{\mathrm{b}}$ & $5.90 \pm 0.01^{\mathrm{b}}$ & $5.84 \pm 0.01^{\mathrm{b}}$ \\
$2^{\text {nd }}$ & $7.64 \pm 0.4^{\mathrm{a}}$ & $5.91 \pm 0.01^{\mathrm{e}}$ & $5.85 \pm 0.02^{\mathrm{e}}$ & $7.47 \pm 0.01^{\mathrm{b}}$ & $6.23 \pm 0.06^{\mathrm{c}}$ & $6.08 \pm 0.08^{\mathrm{d}}$ \\
$3^{\text {rd }}$ & Spoiled & $7.59 \pm 0.04^{\mathrm{b}}$ & $7.41 \pm 0.05^{\mathrm{c}}$ & $8.69 \pm 0.008^{\mathrm{a}}$ & $7.59 \pm 0.03^{\mathrm{b}}$ & $7.58 \pm 0.03^{\mathrm{b}}$ \\
$4^{\text {th }}$ & - & $7.99 \pm 0.01^{\mathrm{b}}$ & $7.95 \pm 0.01^{\mathrm{c}}$ & $8.95 \pm 0.004^{\mathrm{a}}$ & $7.84 \pm 0.01^{\mathrm{d}}$ & $7.83 \pm 0.01^{\mathrm{d}}$ \\
$5^{\text {th }}$ & - & Spoiled & $8.92 \pm 0.13^{\mathrm{a}}$ & Spoiled & $9.14 \pm 0.09^{\mathrm{a}}$ & $8.20 \pm 0.34^{\mathrm{b}}$ \\
$6^{\text {th }}$ & - & - & 9.41 & - & Spoiled & Spoiled \\
\hline
\end{tabular}

The values represent mean \pm SD of three experiments. Means within a row followed by different letters are significantly different $(P<0.05)$ 
Table (3): The effects of various concentrations of natural extracts on Enterobacteriacea Count $(\log$. cfu $/ \mathrm{g})$ of the examined minced meat samples during cold storage at $4 \pm 1{ }^{\circ} \mathrm{C}$.

\begin{tabular}{rcccccc}
\hline Days & Control & $\begin{array}{c}\text { Green tea } \\
(700 \mathrm{ppm})\end{array}$ & $\begin{array}{c}\text { Green tea } \\
(1000 \mathrm{ppm})\end{array}$ & $\begin{array}{c}\text { rosemary } \\
(0.2 \%)\end{array}$ & $\begin{array}{c}\text { Mixture of } \\
(500 \& 0.2 \%)\end{array}$ & $\begin{array}{c}\text { Mixture of } \\
(500 \& 0.3 \%)\end{array}$ \\
\hline Zero & $3.59 \pm 0.11^{\mathrm{b}}$ & $3.25 \pm 0.24^{\mathrm{c}}$ & $3.10 \pm 0.17^{\mathrm{c}}$ & $4.25 \pm 0.02 \mathrm{a}^{\mathrm{a}}$ & $3.84 \pm 0.06^{\mathrm{b}}$ & $3.1 \pm 0.17^{\mathrm{c}}$ \\
$1^{\text {st }}$ & $4.04 \pm 0.03^{\mathrm{b}}$ & $3.93 \pm 0.02^{\mathrm{b}}$ & $3.25 \pm 0.24^{\mathrm{c}}$ & $4.30 \pm 0.02^{\mathrm{a}}$ & $3.99 \pm 0.04^{\mathrm{b}}$ & $3.99 \pm 0.04^{\mathrm{b}}$ \\
$2^{\text {nd }}$ & $5.39 \pm 0.01^{\mathrm{b}}$ & $5.14 \pm 0.03^{\mathrm{c}}$ & $5.14 \pm 0.03^{\mathrm{c}}$ & $5.17 \pm 0.02^{\mathrm{c}}$ & $5.56 \pm 0.01^{\mathrm{a}}$ & $5.41 \pm 0.01^{\mathrm{b}}$ \\
$3^{\text {rd }}$ & - & $6.22 \pm 0.02^{\mathrm{b}}$ & $5.69 \pm 0.08^{\mathrm{c}}$ & $6.20 \pm 0.02^{\mathrm{b}}$ & $6.30 \pm 0.02^{\mathrm{a}}$ & $6.30 \pm 0.02^{\mathrm{a}}$ \\
$4^{\text {th }}$ & - & $6.76 \pm 0.007^{\mathrm{a}}$ & $6.43 \pm 0.01^{\mathrm{c}}$ & $6.69 \pm 0.008^{\mathrm{b}}$ & $6.77 \pm 0.007^{\mathrm{a}}$ & $6.77 \pm 0.007^{\mathrm{a}}$ \\
$5^{\text {th }}$ & - & Spoiled & $6.99 \pm 0.04^{\mathrm{c}}$ & Spoiled & $7.74 \pm 0.007^{\mathrm{a}}$ & $7.54 \pm 0.01^{\mathrm{b}}$ \\
$6^{\text {th }}$ & - & - & 7.47 & - & Spoiled & Spoiled \\
\hline
\end{tabular}

The values represent mean \pm SD of three experiments. Means within a row followed by different letters are significantly different $(P<0.05)$

Table (4): The effects of various concentrations of natural extracts on Staphylococci Count $(\log$. $\mathrm{cfu} / \mathrm{g})$ of the examined minced meat samples during cold storage at $4 \pm 1{ }^{\circ} \mathrm{C}$.

\begin{tabular}{rcccccc}
\hline Days & Control & $\begin{array}{c}\text { Green tea } \\
(700 \mathrm{ppm})\end{array}$ & $\begin{array}{c}\text { Green tea } \\
(1000 \mathrm{ppm})\end{array}$ & $\begin{array}{c}\text { rosemary } \\
(0.2 \%)\end{array}$ & $\begin{array}{c}\text { Mixture of } \\
(500 \& 0.2 \%)\end{array}$ & $\begin{array}{c}\text { Mixture of } \\
(500 \& 0.3 \%)\end{array}$ \\
\hline Zero & $4.57 \pm 0.02^{\mathrm{a}}$ & $4.56 \pm 0.03^{\mathrm{a}}$ & $4.56 \pm 0.01^{\mathrm{a}}$ & $3.81 \pm 0.006^{\mathrm{c}}$ & $4.55 \pm 0.01^{\mathrm{a}}$ & $4.36 \pm 0.05^{\mathrm{b}}$ \\
$1^{\text {st }}$ & $4.73 \pm 0.02^{\mathrm{a}}$ & $4.62 \pm 0.02^{\mathrm{b}}$ & $4.44 \pm 0.03^{\mathrm{c}}$ & $3.85 \pm 0.006^{\mathrm{e}}$ & $4.30 \pm 0.05^{\mathrm{d}}$ & $4.27 \pm 0.03^{\mathrm{d}}$ \\
$2^{\text {nd }}$ & $5.57 \pm 0.02^{\mathrm{a}}$ & $4.86 \pm 0.02^{\mathrm{b}}$ & $4.67 \pm 0.02^{\mathrm{c}}$ & $4.47 \pm 0.01^{\mathrm{e}}$ & $4.67 \pm 0.01^{\mathrm{c}}$ & $4.53 \pm 0.02^{\mathrm{d}}$ \\
3 rd & Spoiled $^{\mathrm{b}}$ & $5.60 \pm 0.02^{\mathrm{a}}$ & $5.54 \pm 0.03^{\mathrm{a}}$ & $4.99 \pm 0.04^{\mathrm{b}}$ & $4.97 \pm 0.11^{\mathrm{b}}$ & $4.96 \pm 0.06^{\mathrm{b}}$ \\
4 th & - & $5.77 \pm 0.007^{\mathrm{a}}$ & $5.59 \pm 0.11^{\mathrm{a}}$ & $5.05 \pm 0.05^{\mathrm{b}}$ & $5.12 \pm 0.13^{\mathrm{b}}$ & $5.10 \pm 0.17^{\mathrm{b}}$ \\
5 th & - & Spoiled & $5.74 \pm 0.01^{\mathrm{a}}$ & Spoiled & $5.15 \pm 0.06^{\mathrm{b}}$ & $5.14 \pm 0.03^{\mathrm{b}}$ \\
$6^{\text {th }}$ & - & - & 6.25 & - & Spoiled & Spoiled \\
\hline
\end{tabular}

The values represent mean \pm SD of three experiments. Means within a row followed by different letters are significantly different $(P<0.05)$

Table (5) Reduction percentage of microbial growth with different concentrations of natural extracts.

- No reduction

\begin{tabular}{lllllllll}
\hline M.O & Groups & zero & $1^{\text {st }}$ & $2^{\text {nd }}$ & $3^{\text {rd }}$ & $4^{\text {th }}$ & $5^{\text {th }}$ & $6^{\text {th }}$ \\
\hline APC & Green tea 700 & $96 \%$ & $71 \%$ & $52 \%$ & - & - & - & - \\
& Green tea 1000 & $97 \%$ & $80 \%$ & $59 \%$ & - & - & - & - \\
& Rosemary $0.2 \%$ & $59 \%$ & $48 \%$ & - & - & - & - & - \\
& mix.(500\&0.2\%) & $96 \%$ & $53 \%$ & - & - & - & - & - \\
& Mix(500\&0.3\% & $97 \%$ & $59 \%$ & $28 \%$ & - & - & - & - \\
\hline Staphylococci & & & & & & & & \\
& Green tea 700 & $1 \%$ & - & - & - & - & - & - \\
& Green tea 1000 & $2 \%$ & $26 \%$ & - & - & - & - & - \\
& Rosemary 0.2\% & $82 \%$ & $80 \%$ & $20 \%$ & - & - & - & - \\
& mix.(500\&0.2\%) & $4 \%$ & $46 \%$ & - & - & - & - & - \\
& Mix(500\&0.3\% & $38 \%$ & $50 \%$ & $9 \%$ & - & - & - & - \\
\hline Enterobacteriacea & & & & & & & & \\
& Green tea 700 7000 & - & - & - & - & & - \\
& Green tea 1000 & $66 \%$ & $50 \%$ & - & - & - & & - \\
& Rosemary 0.2\% & - & - & - & - & - & - \\
& mix.(500\&0.2\%) & - & - & - & - & & - \\
& Mix(500\&0.3\% & $66 \%$ & - & - & - & & - \\
\hline
\end{tabular}


Table (6): The effects of various concentrations of natural extracts on $\mathrm{pH}$ of the examined minced meat samples stored at $4 \pm 1{ }^{\circ} \mathrm{C}$.

\begin{tabular}{ccccccc}
\hline Days & Control & $\begin{array}{c}\text { Green tea } \\
(700 \mathrm{ppm})\end{array}$ & $\begin{array}{c}\text { Green tea } \\
(1000 \mathrm{ppm})\end{array}$ & $\begin{array}{c}\text { rosemary } \\
(0.2 \%)\end{array}$ & $\begin{array}{c}\text { Mixture of } \\
(500 \& 0.2 \%\end{array}$ & $\begin{array}{c}\text { Mixture of } \\
(500 \& 0.3 \%\end{array}$ \\
\hline Zero & $5.9 \pm 0.12^{\mathrm{b}}$ & $6.2 \pm 0.3^{\mathrm{ab}}$ & $6.3 \pm 0.10^{\mathrm{ab}}$ & $6.2 \pm 0.2^{\mathrm{ab}}$ & $6.1 \pm 0.5^{\mathrm{ab}}$ & $6.5 \pm 0.14^{\mathrm{a}}$ \\
$1^{\text {st }}$ & $6.0 \pm 0.2^{\mathrm{a}}$ & $6.2 \pm 0.10^{\mathrm{a}}$ & $6.3 \pm 0.4^{\mathrm{a}}$ & $6.4 \pm 0.13^{\mathrm{a}}$ & $6.1 \pm 0.2^{\mathrm{a}}$ & $6.3 \pm 0.12^{\mathrm{a}}$ \\
$2^{\text {nd }}$ & $6.0 \pm 0.14^{\mathrm{b}}$ & $6.2 \pm 0.3^{\mathrm{ab}}$ & $6.3 \pm 0.12^{\mathrm{ab}}$ & $6.5 \pm 0.23^{\mathrm{a}}$ & $6.2 \pm 0.21^{\mathrm{ab}}$ & $6.3 \pm 0.10^{\mathrm{ab}}$ \\
$3^{\text {rd }}$ & Spoiled & $5.9 \pm .20^{\mathrm{b}}$ & $6.1 \pm 0.11^{\mathrm{ab}}$ & $6.2 \pm 0.4^{\mathrm{ab}}$ & $6.0 \pm 0.14^{\mathrm{ab}}$ & $6.4 \pm 0.22^{\mathrm{a}}$ \\
$4^{\text {th }}$ & - & $5.8 \pm 0.1^{\mathrm{a}}$ & $5.9 \pm 0.3^{\mathrm{a}}$ & $6.0 \pm 0.2^{\mathrm{a}}$ & $5.9 \pm 0.2^{\mathrm{a}}$ & $6.1 \pm 0.1^{\mathrm{a}}$ \\
$5^{\text {th }}$ & - & Spoiled & $5.9 \pm 0.4^{\mathrm{a}}$ & - & $5.8 \pm 0.1^{\mathrm{a}}$ & $5.8 \pm 0.2^{\mathrm{a}}$ \\
$6^{\text {th }}$ & - & - & 5.8 & - & - & - \\
\hline
\end{tabular}

Means within a row followed by different letters are significantly different $(P<0.05)$. The values represent mean $\pm \mathrm{SD}$ of three experiments.

Table (7): The effects of various concentrations of natural extracts on TBA (Melanoaldehyde $/ \mathrm{Kg}$ ) of the examined minced meat samples during cold storage at $4 \pm 1^{\circ} \mathrm{C}$.

\begin{tabular}{ccccccc}
\hline Days & Control & $\begin{array}{c}\text { Green tea } \\
(700 \mathrm{ppm})\end{array}$ & $\begin{array}{c}\text { Green tea } \\
(1000 \mathrm{ppm})\end{array}$ & $\begin{array}{c}\text { rosemary } \\
(0.2 \%)\end{array}$ & $\begin{array}{c}\text { Mixture of } \\
(500 \& 0.2 \%)\end{array}$ & $\begin{array}{c}\text { Mixture of } \\
(500 \& 0.3 \%)\end{array}$ \\
\hline Zero & $0.78 \pm 0.02^{\mathrm{a}}$ & $0.71 \pm 0.01^{\mathrm{b}}$ & $0.63 \pm 0.03^{\mathrm{c}}$ & $0.54 \pm 0.01^{\mathrm{e}}$ & $0.70 \pm 0.02^{\mathrm{b}}$ & $0.59 \pm 0.01^{\mathrm{d}}$ \\
$1^{\text {nd }}$ & $0.84 \pm 0.04^{\mathrm{a}}$ & $0.72 \pm 0.01^{\mathrm{b}}$ & $0.69 \pm 0.02^{\mathrm{b}}$ & $0.60 \pm 0.01^{\mathrm{c}}$ & $0.71 \pm 0.01^{\mathrm{b}}$ & $0.69 \pm 0.03^{\mathrm{b}}$ \\
$2^{\text {nd }}$ & $0.86 \pm 0.01^{\mathrm{a}}$ & $0.74 \pm 0.02^{\mathrm{b}}$ & $0.70 \pm 0.04^{\mathrm{b}}$ & $0.64 \pm 0.01^{\mathrm{c}}$ & $0.72 \pm 0.02^{\mathrm{b}}$ & $0.71 \pm 0.01^{\mathrm{b}}$ \\
$3^{\text {rd }}$ & Spoiled $^{\mathrm{a}}$ & $0.79 \pm 0.03^{\mathrm{ab}}$ & $0.75 \pm 0.01^{\mathrm{bc}}$ & $0.69 \pm 0.01^{\mathrm{d}}$ & $0.81 \pm 0.01^{\mathrm{a}}$ & $0.72 \pm 0.04^{\mathrm{cd}}$ \\
$4^{\text {th }}$ & - & $0.83 \pm 0.02^{\mathrm{ab}}$ & $0.81 \pm 0.01^{\mathrm{a}}$ & $0.79 \pm 0.05^{\mathrm{b}}$ & $0.87 \pm 0.02^{\mathrm{a}}$ & $0.79 \pm 0.02^{\mathrm{b}}$ \\
$5^{\text {th }}$ & - & Spoiled & $0.81 \pm 0.01^{\mathrm{b}}$ & Spoiled & $0.89 \pm 0.02^{\mathrm{a}}$ & $0.81 \pm 0.03^{\mathrm{b}}$ \\
$6^{\text {th }}$ & - & - & 0.83 & - & Spoiled & Spoiled \\
\hline
\end{tabular}

Table (8): The effects of various concentrations of natural extracts on TVN $(\mathrm{mg} / 100 \mathrm{~g})$ of the examined minced meat samples during cold storage at $4 \pm 1{ }^{\circ} \mathrm{C}$.

\begin{tabular}{rrccccc}
\hline Days & Control & $\begin{array}{c}\text { Green tea } \\
(700 \mathrm{ppm})\end{array}$ & $\begin{array}{c}\text { Green tea } \\
(1000 \mathrm{ppm})\end{array}$ & $\begin{array}{c}\text { rosemary } \\
(0.2 \%)\end{array}$ & $\begin{array}{c}\text { Mixture of } \\
(500 \& 0.2 \%)\end{array}$ & $\begin{array}{c}\text { Mixture of } \\
(500 \& .03 \%)\end{array}$ \\
\hline Zero & $13.9 \pm 0.13^{\mathrm{a}}$ & $11.8 \pm 0.4^{\text {cd }}$ & $11.4 \pm 0.2^{\mathrm{d}}$ & $12.1 \pm 0.11^{\mathrm{bc}}$ & $12.5 \pm 0.3^{\mathrm{b}}$ & $11.9 \pm 0.1^{\mathrm{c}}$ \\
$1^{\text {st }}$ & $14.1 \pm 0.2^{\mathrm{a}}$ & $12.1 \pm 0.12^{\mathrm{c}}$ & $11.9 \pm 0.3^{\mathrm{c}}$ & $12.3 \pm 0.2^{\mathrm{c}}$ & $12.9 \pm 0.5^{\mathrm{b}}$ & $12.0 \pm 0.10^{\mathrm{c}}$ \\
$2^{\text {nd }}$ & $14.3 \pm 0.10^{\mathrm{a}}$ & $12.5 \pm 0.3^{\mathrm{c}}$ & $12.4 \pm 0.14^{\mathrm{c}}$ & $12.3 \pm 0.3^{\mathrm{c}}$ & $13.1 \pm 0.5^{\mathrm{b}}$ & $12.3 \pm 0.1^{\mathrm{c}}$ \\
$3^{\text {rd }}$ & Spoiled & $14.0 \pm 0.4^{\mathrm{a}}$ & $13.0 \pm 0.2^{\mathrm{bc}}$ & $12.5 \pm 0.10^{\mathrm{d}}$ & $13.4 \pm 0.3^{\mathrm{b}}$ & $12.9 \pm 0.1^{\mathrm{c}}$ \\
$4^{\text {th }}$ & - & $14.4 \pm 0.1^{\mathrm{a}}$ & $13.5 \pm 0.3^{\mathrm{b}}$ & $12.6 \pm 0.13^{\mathrm{d}}$ & $13.5 \pm 0.2^{\mathrm{b}}$ & $13.1 \pm 0.10^{\mathrm{c}}$ \\
$5^{\text {th }}$ & - & Spoiled & $13.6 \pm 0.10^{\mathrm{a}}$ & Spoiled & $13.8 \pm 0.5^{\mathrm{a}}$ & $13.8 \pm 0.15^{\mathrm{a}}$ \\
$6^{\text {th }}$ & - & - & 14.9 & - & Spoiled & Spoiled \\
\hline
\end{tabular}

The values represent mean $\pm \mathrm{SD}$ of three experiments. Means within a row followed by different letters are significantly different $(P<0.05)$

\section{DISSUCSION:}

Sensory profile allowed us to evaluate the quality of food and in some time to identify unwanted contaminants (Rasooli, 2007). It is obvious from results obtained in table (1) that the sensory properties of different treated minced meat samples during cold storage $\left(4 \pm 1^{\circ} \mathrm{C}\right)$ were enhanced and shelf-life were extended by increasing the concentration of extracts compared to the untreated (control) ones. Generally, samples containing green tea (1000 ppm) demonstrated the highest enhancement of sensory attributes and longest shelf-life then followed by mixture of green tea extract $(500 \mathrm{ppm}) \&$ rosemary extract $(0.3 \%)$ and mixture of green tea extract $(500 \mathrm{ppm}) \&$ rosemary extract $(0.2 \%)$ then green tea extract $(700$ $\mathrm{ppm})$ and rosemary extract $(0.2 \%)$. This result agree with (Ucak et al., 2011) and (Pateiro et al., 2015) who recorded that green tea extract lower total color difference between 0 and 24 weeks of refrigerated stored pig pâtés. 
The results illustrated in table (2) were agree with those recorded by (Kang et al., 2005) who found that APC of control samples were the highest and followed by those coated with pectin-based materials containing $0.5 \%$ green tea powder (CGP). Also, (Baker et al., 2013) who found that rosemary extract decrease APC as it recorded (11.50 \pm 0.28$)(\log . \mathrm{cfu} / \mathrm{g})$ while control recorded (39.00 \pm 0.57$)(\log . \mathrm{cfu} / \mathrm{g})$. The control samples were unaccepted at zero day while treated ones with green tea extract $(1000 \mathrm{ppm})$, green tea extract $(700 \mathrm{ppm})$, mixture of green tea extract $(500 \mathrm{ppm}) \&$ rosemary extract $(0.2 \%)$ and mixture of green tea extract $(500 \mathrm{ppm}) \&$ rosemary extract $(0.3 \%)$ remained accepted untill $2^{\text {nd }}$ day of storage, according to (EOS, 2005) as permissible limit $\left(10^{6}\right)$.

Also, Enterobacteriaceae count (log. cfu/g) decrease and this result agree with (Gai et al., 2014) who found that green tea decrease Enterobacteriaceae count of fillets samples compared to control sample, as green tea recorded "2.31" (log. cfu/g) while control "2.68" (log. cfu/g). (table 3). As shown in table (4), the control samples had the highest counts of Staphylococcus count and this nearly similar to those obtained by(Abramovič et al., 2012) who found that rosemary extract has strong antibacterial activity especially against gram-positive bacteria by use broth microdilution test using minimal inhibitory (MIC) concentrations in the range of 0.02 to $0.06 \mathrm{mg} / \mathrm{mL}$ and (Alzoubi et al., 2014) who found that rosemary extract has antibacterial activity against MRSA by use Fifty micro-liters of $100 \mathrm{mg} / \mathrm{ml}$ of $\mathrm{R}$. officinalis showed the greater effect with $\mathrm{MIC}$ and $\mathrm{MBC}$ values range of $0.125-0.25 \mathrm{mg} / \mathrm{ml}$ and $0.25-0.5 \mathrm{mg} / \mathrm{ml}$. The control as well as all treated groups were unaccepted by (EOS, 2005) as permissible limit of Staphylococci in minced meat $\left(10^{2}\right) . \mathrm{pH}$ plays an important role for microbiological growth affecting shelf-life of the meat products. (Amal and Soher, 2010). Table (6) showed $\mathrm{pH}$ mean values those agree with (Faisal, 2011) who found that green tea extract had higher $\mathrm{pH}$ values (5.83) compared to control samples (5.69)., This may due to green tea extract $\mathrm{pH}$ close to (7.70) as reported by (KobusCisoweka et al., 2010).

At the same time, TBA is a good indicator for the assessment of quality of meat and degree of lipid oxidation. (Ndaw et al., 2008). This result agree with (Faisal, 2011) who found that green tea extract lowered the peroxide values $(2.35 \pm 0.29)$ compared to control samples $(6.48 \pm 1.89)$. The increased in TBA value may be due to lipid hydrolysis and secondary products formation under low temperature. (Forrest et al., 1975). According to (EOS, 2005) the TBA value accepted till $0.9 \mathrm{mg}$
Melanoaldehyde / Kg raw minced beef. All samples were accepted.

Moreover, TVN measurement is the traditional chemical mean most widely used for evaluation of degree of meat spoilage. The obtained results agree with (Baker et al., 2013) who found that addition of rosemary with or without sodium lactate resulted in lower values of TVN as compared to control. The increase in TVN might be due to microbial activity under low temperature. Ibrahim and Desouky, (2009), also may be attributed to the breakdown of proteins as a result of activity of microbial strains and proteolytic enzymes.(Yassin-Nessrien, 2003). The TVN value accepted till $20 \mathrm{mg} / 100 \mathrm{~g}$ in raw minced beef. All the examined samples of both treated and untreated groups were accepted according to (EOS, 2005).

In conclusion, rosemary and green tea extracts have both of antimicrobial and antioxidant effects. Green tea extract proved to be more efficient than rosemary extract especially green tea extract (1000 ppm). So, the use of natural extracts as it is safe natural agent, is therefore recommended to improve safety and extend shelf life of meat product.

\section{REFERENCES}

Abramovič, H., Terpinc, P., Generalić, I., Skroza, D., Klančnik, A., Katalinić, V., Možina, S.S., 2012. Antioxidant and antimicrobial activity of extracts obtained from rosemary (Rosmarinus officinalis) and vine (Vitis vinifera) leaves Croat. J. Food Sci. Technol. 4: 1-8.

Ahmed, I.M.I., 1991. Hygienic quality of marketed ready to eat meat. M.V.Sc. Thesis Meat hygiene, Fac. Vet. Med.; Zagazig Univ. .

Alzoubi, H.M., Ibrahim, A.I., Alsbou, M.S., Aqel , A.A., 2014. Inhibitory effect of mediterranean sage and Rosemary on clinical and community isolates of Methicillin-Resistant Staphylococcus aureus. Jordan J of Biological Sci 7: 161-164.

Amal, S.H., Soher, E.A., 2010. Role of lactic acid bacteria as a biopreservative agent of Talbina. $\mathrm{J}$ of American Sci 6: 889-898. .

Baker, I.A., Alkass, J.E., Saleh, H.H., 2013. Reduction of oxidative rancidity and microbial activities of the karadi lamb patties in freezing storage using natural antioxidant extracts of rosemary and ginger. International J of Agricultural and Food Research 2: 31-42.

Biesalski, H.K., 2005. Meat as a component of a healthy diet - are there any risks or benefits if meat is avoided in the diet? Meat science 70: 509-524.

Del Campo, J., Amiot, M.J., Nguyen-The, C., 2000. Antimicrobial effect of rosemary extracts. Journal of food protection 63: 1359-1368. 
EOS, 2005. (Egyptian Organization for Standardization and Quality 2005. Egyptian Standard, ES.

Faisal, A.M., 2011. Effects of Green Tea extract on color and Lipid oxidation in Ground Beef Meat. J Tikrit Univ. For Agri. Sci. 13, 351-354.

FAO, 1980. Food and Agriculture Organization. Manual of food quality control. 4. Microbiological Analysis, FAO, United Nations, Rome. Int. J. Food Microbiol., 80, 241-250.

Forrest, J.C., Aberle, E.D., Hedrick, H.B., Judage, M.D., Merkel, R.A., 1975. Principles of meat science, WH Freeman, San Francisco, CA, . 240-248.

Gai, F., Gasco, L., Ortoffi, M., Gonz ales-Rodriguez , A., Parisi, G., 2014. Effects of green tea natural extract on quality parameters and lipid oxidation during storage of tench (Tinca tinca) fillets. J. Appl. Ichthyol 30: 64-71.

Gomez, K.A., Gomez, A.A., 1984. Statistical procedures for agriculture research. . John Wiliy and Sons Editor Inc. USA (2Ed.), Chapter 3: 129-184. .

Gork, E.P., 1976. Uber die Ursachen von Qualitatstmangeln bei tiefgeforenen Fertiggerichten auf fleischbasis in der Fluggastverpflegung. . Vet. Med. Diss., tech. Univ. Berlin.

Gramza, A., Korczak, J., Hes, M., Jedrusekgolinska, A., 2004. Tea extracts influence on catalytical properties of $\mathrm{Fe} 2+$ in lipids. Pol. J. Environ. Studies, 13: 143.

Ibrahim, S.M., Desouky, S.G., 2009. Effect of antimicrobial metabolites produced by lactic acid bacteria on quality aspects of frozen Tilipia (Oreochromis niloticus) fillets. World J . of Fish and Marine Sciences, 1: 40-45.

ICMSF, 1978. International Commission and Microbiological Specification for Food. Microorganism in foods. Their significance and methods of enumeration. 1st, 2nd Ed Toronto, Univ. of Toronto press. Toronto Ontario, . Canada. J.Hyg., 33: 165-174.

ICMSF., 1996. International Commission and Microbiological Specification for Food Microorganism in foods 1.Their significance and methods of enumeration. 3rd Ed Toronto, Univ. of Toronto press.

Kang, H.J., Jo, C., Kwon, J.H., Kim, J.H., Chung, H.J., Byun, M.W., 2005. Effect of a pectin- based edible coating containing green tea powder on the quality of irradiated pork patty. Food Control 18: 430-435.

Kobus-Cisoweka, J., Flaczyk, E., Jeszka, M., 2010. Antioxidants activities of Ginkgo biloba extract: application of freeze stored meat dumphlings. Acta Sci. Pol., Technol Aliment 9: 161-170.

Koutsoumanis, K., Giannakourou, M.C., Taoukis, P.S., Nychas, G.J., 2002. Application of shelf life decision system (SLDS) to marine cultured fish quality. International journal of food microbiology 73: 375-382.
Kristanti, R.A., Punbusayakul, N., 2009. Antioxidant and antimicrobial activity of commercial green tea in Chiang Rai. Acta Horticultura J, 53-58.

Lambert, A.D., Smith, J.P., Dodds, K.L., 1991. Shelf life extension and microbiological safety of fresh meat. A review. Food Microbiol. 9: 267-297.

Mercuri, A.J., Cox, N.A., Carson, M.O., Tanner, D.A., 1978. Relation of Enterobacteriaceae count to Salmonella contamination of Marker broiler. J. Food Prot. 42: 427.

Mielnik, M.B., Signe, S., Bjrg, E., Grete, S., 2008. Byproducts from herbs essential oil production as ingredient in marinade for turkey thighs. LWT 41: 93-100.

Ndaw, A.D., Faid, M., Bouseta, A., Zinedine, A., 2008. Effect of controlled lactic acid bacteria fermentation on microbiological and chemical quality of Morocans sardines (Sardina pilchardus). Int. J. of Agric. and biology 10: 2127.

Olaoye, O.A., Onilude, A.A., 2010. Investigation on potential use of biological agents in the extension of fresh beef in Nigeria. . World J of Microbiol. and biotechnol. 26: 1445-1424.

Pateiro, M., Lorenzo, J.M., Vazquez, J.A., Franco, D., 2015. Oxidation Stability of Pig Liver Pate with Increasing Levels of Natural Antioxidants (Grape and Tea). Antioxidants (Basel, Switzerland) 4: 102-123.

Pearson, D., Tauber, 1984. Chemical Analysis of Food. 8th Ed. Publishing Co. Churchill Livingstone,s, Edinburgh, London, UK.

Rasooli, I., 2007. Food preservation-A biopreserrvative approach. Global Science Books, Food, 1: 111136.

Ucak, I., Ozogul, Y., Durmus, M., 2011. The effects of rosemary extract combination with vacuum packing on the quality changes of Atlantic mackerel fish burgers. Int. J of Food Sci. and Technol. 46: 1157-1163.

Vyncke, W., 1970. Direct determination of thiobarbituric acid value in trichloroacetic acid extracts of fish as a measure of oxidative rancidity. Fette Seifen Anstri -climitted 72: 1084-1087.

Yassin-Nessrien, M.N., 2003. Effect of storage conditions on the quality parameters of differently treated fish. Ph. D. Thesis, Fac. Agric. Ain Shams, Univ. Cairo. Egypt. 